\title{
TECCIENCIA
}

\section{IPTV: next-generation network technologies and protocols}

\section{IPTV: tecnologías y protocolos orientados a redes de próxima generación}

\author{
Danilo López ${ }^{1}$, Bayron Villanueva ${ }^{2}$, Edwin Rivas ${ }^{3}$ \\ ${ }^{1}$ Universidad Distrital “Francisco José de caldas”, Bogotá, Colombia, dalopezs@ udistrital.edu.co \\ ${ }^{2}$ Universidad Distrital "Francisco José de caldas", Bogotá, Colombia, bfv88@ hotmail.com \\ ${ }^{3}$ Universidad Distrital "Francisco José de caldas”, Bogotá, Colombia, erivas@udistrital.edu.co
}

\begin{abstract}
Television has been one of the most important media used by man since its inception. It is, therefore, essential nowadays to find a method to optimize the transmission process and interaction with the end user. The widely used conventional transmission system is inefficient in many respects, taking into account the means of transmission and the lack of customization of content for users. Therefore, due to the steady growth and development of the Internet and IP-based information technologies, IP networks for the delivery of television content live and on-demand have begun to be used, where the user plays an active role.
\end{abstract}

Keywords: Compression, IMS, IPTV, Multicast, Streaming.

\section{Resumen}

La televisión ha sido uno de los medios de comunicación más importantes y utilizados por el hombre desde su creación; es por eso que buscar un método de optimizar el proceso de transmisión e interactuación con el usuario final resulta indispensable en la actualidad. El sistema de transmisión convencional, ampliamente utilizado, resulta ineficiente en muchos aspectos, teniendo en cuenta los medios de transmisión y la falta de personalización de contenidos para los usuarios. Por lo cual, gracias al constante crecimiento y desarrollo de la Internet y las tecnologías de la información basadas en IP, se ha comenzado a utilizar las redes IP para la entrega de contenidos televisivos en vivo y bajo demanda, donde el usuario forma parte activa de ésta.

Palabras claves: Compresión, IMS, IPTV, Multicast, Streaming

\section{Introduction}

Due to the Internet's rapid development, research and development in communication networks have benefited with the development of a set of new protocols and technologies, allowing wider bandwidths, faster and more efficient communication technologies, precise real-time management systems, and innovative coding schemes and protocols. This progress allowed the emergence of a new host of services ranging from the appearance of private virtual networks [1], the VoIP technologies [2], to media streaming over packet networks and the emergence of Internet Protocol Television (IPTV).
The IPTV is multimedia software that has gained strength in recent years and is being applied and successfully marketed worldwide. IPTV is a way of delivering digital TV signal through the use of IP networks to reach users. The ITU-T [3] defines it as: "a set of multimedia services like television/video/audio/text/graphics/data that are transported over IP-based networks and managed to provide reasonable levels of quality of service, security, interactivity, and trust".

What differentiates IPTV service from traditional TV service is the ability to raise the user's entertainment experience providing a range of 


\section{TECCIENCIA}

additional services that make the user not only a simple consumer of content, but also player (providing interaction with the content and service by taking advantage of the two-way communication capability of IP networks) and possibly also a content creator (enabling users to share content created or edited by them with other users [4]), providing in a similar manner the services that suppliers of digital cable or satellite television are already providing in order to be competitive.

For companies involved in providing telecommunications services, the IPTV service becomes very attractive because it allows them to compete with companies providing triple play services (TV, telephony, and data) via cable or satellite, allowing them to create their own triple or quadruple play service (including mobile services) providing data, voice (VoIP), and television (IPTV) on the communication infrastructure they have already implemented.

The idea of transmitting television via data networks is not new [5], in fact, this idea came up in the 90s, but at that time it was not very successful because the technology available (ATM, DSL, MPEG) was not sufficiently well developed, besides being too expensive to reap the benefits. Today, this is no longer a problem; the cost of technology has dropped dramatically and it has evolved to the point that compression methods are far more efficient (MPEG4 part 10), the technology to reach the user handles wider bandwidths (ADSL2, VDSL, FTTH), and very high capacity IP packet routers have been developed.

We must not confuse the IPTV service with the socalled Internet TV with which most people are familiar. Internet $\mathrm{TV}$ is defined as any video delivered over the public Internet to users through the IP core network that supports the Internet [6]; therefore, because the Internet is a "best effort" network, it does not provide criteria for quality of Service (QoS) nor does it guarantee a minimum quality of experience (QoE) to users, which can degrade the signal quality at times of high congestion. In contrast to Internet TV, IPTV is deployed over private networks managed by providers of telecommunications services, using QoS criteria and guaranteeing a QoE that competes with or improves the quality provided by other broadcast TV services.

This article focuses on studying the state-of-the-art in the main technologies and innovations in the field of IPTV with emphasis on the most innovative technologies that due to technological development (IPv6, MPLS, and H.264) allow for the design of a more powerful and efficient transmission platform. Section II recaps IPTV by reviewing its main components, players, and architectures, as well as the benefits and services it provides. Section III provides an overview of the main technologies of multimedia content compression with special focus on H.264/AVC. Section IV reviews the main transport protocols used in the IPTV service (RTP, RTSP, and RTCP) and Multicasting protocols used in IPv6 data networks. In Section V, we review the IPTV architecture based on the IMS system, its main components, protocols, and advantages. Finally, the conclusions and future work are in Section VI.

\section{IPTV Components architecture and operation}

\subsection{Overview}

By studying the IPTV service, their value chain can be identified consisting of four elements [7]: (1) Production of contents, where different contents (movies, series, documentaries...), are created and edited; (2) Aggregation of content, which brings together the content from various sources and these are offered to customers; (3) Content delivery, which is responsible for transporting the contents in encrypted form to customers or end users; (4) Reconstitution of content, transforming the encrypted content to be suitable for viewing by consumers.

In this chain there are four players who influence it: (1) Content Provider who owns the rights to the content produced; (2) the Service Provider, which is a telecommunications operator responsible for providing various services to consumers; (3) the Network Provider is the organization that maintains and operates the network components required for IPTV functionality; (4) End User who is the consumer of IPTV services.

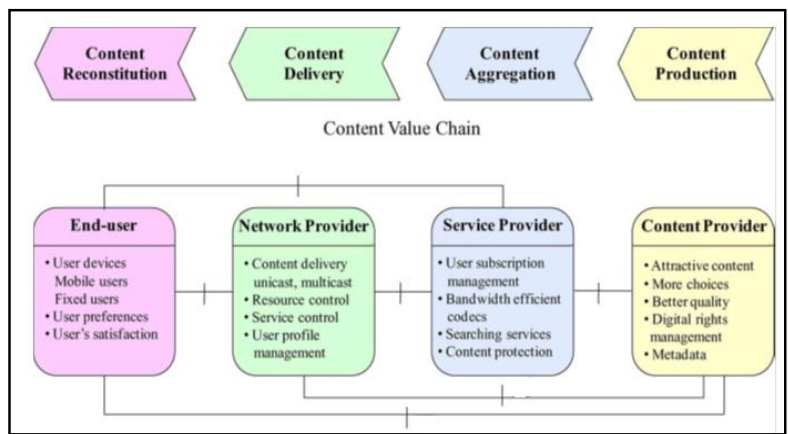

Figure 1. IPTV value chain [7]

IPTV can offer many types of services [5] [6] [7], the main one being real-time TV service, which is well-known to everybody and is similar to the broadcast TV service, with land or cable transmission. Another main service in any IPTV 
system is the Video on Demand (VoD) service where the authorized user has access to certain prerecorded content and has total control over the reproduction, being able to pause, fast forward, or rewind the content at any given time.

Besides these two main services, there are a set of ancillary services including, for example, the electronic program guide (EPG), which allows users to view different contents grouped into TV channels or VoD channels, with their description and schedule; a personnel recorder service (PRS) is also available that stores real-time broadcast content for later viewing and, likewise, can provide interactive services allowing users to interact with content in real time with other users or produce new content.

\subsection{Standardization}

There are currently various entities responsible for the standardization of the IPTV service, although most began their work independently, several of these entities are currently working together to define a single standard that facilitates the compatibility among devices and services in the industry.

The ITU-T [2] formed a working group called Focus Group on IPTV (FG IPTV) with the mission of coordinating and developing a standard architecture based on a client-server model with the addition of a service delivery platform. ETSIVTISPAN [8] has the mission of developing specifications for Next Generation Networks (NGN), defining IPTV as a NGN service and using IP Multimedia Subsystem (IMS) the standard created by 3GPP [9]. Open IPTV Forum [10] works in conjunction with other standardization groups to define end-to-end delivery of IPTV specifications.

A great effort is being made by the ITU-T in conjunction with ETSI to develop an IPTV service architecture that integrates the use of NGN [11]. The ITU-T defines NGN as a packet-based network able to provide telecommunication services and use multiple bandwidths and transportation technologies that implement QoS in which service-related functions and control over it are independent of transport technologies that support the lower layers.

\subsection{Architectures}

Three types of architectures have been formally defined by the ITU-T [6] [7]. First, there is the nonNGN IPTV architecture that is based entirely on existing components and network protocols, which requires monitoring of services and an application layer, separated and developed specifically to handle
IPTV services. The second is the NGN IPTV architecture, which uses components of the NGN Functional Architecture [12] standardized by the ITU-T to support the provision of IPTV services. Finally, the IPTV NGN architecture based IMS, which is an architectural subsystem that provides and controls multimedia services on the NGN core network; due to this, the system provides signaling, management and authorization of users in standardized manner.

\subsection{Components}

When analyzing the technical infrastructure of an IPTV platform, three main components can be recognized (Figure 1); these are the video head end, the transport network and the end user. In [13] we can see a complete overhaul of the infrastructure entirely from a technical standpoint.

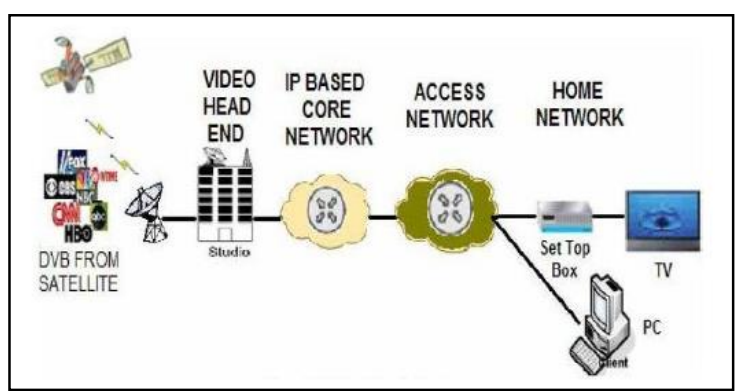

Figure 2. IPTV end-to-end System Components

[14]

In the header of the video, linear and on-demand contents are captured and formatted for distribution over IP networks [6]. It consists of a video encoder that captures analog video signals sent by the content distributor and transforms them into a compressed digital format suitable for transmission over packet networks; a real-time video server, responsible for transmitting the video streams using a real-time transmission protocol (RTP) on the network that uses multicasting techniques; a server for video on demand with great storage capacity to store the various contents provided to customers and sends the video streams in real time (with the ability to control playback) using unicasting on the transport network.

The transportation network is made up of two parts, the core network or nucleus of the network and the access network. The core network is basically a set of routing devices that manage the IP protocol and implement different transport techniques, multicasting and QoS control. MPLS [15] is a data transport mechanism that improves traffic engineering [14]; with the use of MPLS IP messages behave as connection-oriented given that the path 


\section{TECCIENCIA}

between a source and a destination is pre-determined and labeled; tags are used to establish end-to-end paths called LSPs.

The access network, also known as "the last mile" is the section of the network that reaches the user by providing broadband connection; a number of technologies are available to deliver connection to the end user (xDSL, FTTx, WiiMAX), which in the case of IPTV should be chosen very carefully in order to deliver adequate bandwidth to provide multiple television channels to a home taking into account that a channel in standard definition requires at least $2 \mathrm{Mbps}$, and for a high-definition (HD) channel it needs at least 9 Mbps using advanced compression techniques [5].

End users must be able to receive the transmitted signal and to decode and adapt it to be displayed on a standard video receiver. Generally, it is a Set-Top Box with functions to decode the compressed video, interpret the meta-data received, and deliver the video signal through some of its output interfaces to the TV.

\subsection{IPTV System Operation}

All contents from one or more content providers reach the video head end. These providers can deliver broadcast content in real time or content to be stored and delivered on demand. The contents arriving at the head of video can be sent by using analog or digital signals in multiple formats, so that the video encoder is responsible for transforming these signals into a standard compressed digital format suitable for distribution and/or storage.

Many compression techniques exist, both free and proprietary, techniques using spatial and/or temporal compression. The most known and used are MPEG2, MPEG4, H.264/AVC (or MPEG4 part10), WMV; the most used currently is MPEG2, which provides high compression (up to 50 times [13]) and uses a relatively simple encoder/decoder that does not consume much processing power; on the other hand, the most efficient is h.264, which has much greater performance than the MPEG2, allowing much higher compression by reducing the bandwidth required to transmit one channel, the drawback is that it employs much more complex encoders/decoders that need much more processing power, but with the high-performance servers of today capable of working in mesh this does not represent a major drawback. H.264 is the compression technique upon which this article focuses, being the most innovative, advanced, and efficient approach.
After the coding stage, on-demand contents are stored in the respective video on-demand servers ready to be transmitted to users who request them; real-time video content (standard television channels) and on-demand already encoded content, are encapsulated into a multimedia stream transport format, usually MPEG2-TS that multiplexes the independent video, audio and data streams provided by the encoder for shipping within a single flow on the transport network.

Subsequently, these MPEG2-TS streams are encapsulated by using the RTP [16] real-time transport protocol that guarantees orderly delivery of packets over data networks based on IP; RTP packets are encapsulated on the UDP [17] protocol instead of the TCP, because TCP makes a signposted establishment connection to ensure delivery of packages; the IPTV service does not need to retransmit a lost packet because it would lose its status as real-time transport; therefore, a nonconnection oriented protocol such as UDP is the most efficient way to deliver content in real time.

For real-time television, the encapsulated packets are sent to users using multicast techniques. Each TV channel is transmitted to a different multicast address and users when choosing the channel they want to watch are directed to the appropriate multicast address to obtain the stream content. Using multicast to transmit video channels in real time leads to optimizing the system bandwidth; each channel is transmitted only once on the routers that communicate with the users requesting the transmission of a channel; all users tuned to the same channel get the same content without the possibility of controlling the flow of video. There are different multicasting protocols to manage the core network, the most used are PIM-SM and PIM-DM, and on the edge of the network multicast group management protocols are needed such as IGMP for IPv4 and ML for DIPv6.

In the case of VoD, transport over the network uses unicasting techniques given that each user must have full control over the playback of its contents; using unicasting causes network congestion to a great extent and it is advisable to implement various VoD servers nearby to users. To transport the contents, RTP on UDP is used, but to control playback, RTSP protocol messages [18] are used; this protocol has different types of messages that tell the system what action the user wants to execute on playing multimedia content (pause, play, forward, rewind). 


\section{Video Compression Techniques}

There are several techniques to compress video, audio, and data, but certainly the most successful have been those developed by the Moving Pictures Experts Group [19], they began with MPEG1 then they developed MPEG2, MPEG4 part 2 and, finally, MPEG4 part 10, better known as h. 264/AVC. Each uses its predecessor's techniques improving them and increasing the compression ratio; the following will provide a brief explanation of MPEG2 and then the operation of H.264 will be explained.

\subsection{MPEG-2}

Videos are nothing but a sequence of images visualized at a sequentially determined rate, each video image is known as a frame and to be visualized seamlessly it must be reproduced at a rate of 25 frames per second (fps) [20].

MPEG2 uses various techniques to compress videos. First, it performs a spatial processing, dividing each frame into horizontal slots 16 pixels wide, each slot in turn is divided into "macro blocks" of $16 \times 16$ pixels, each macro block is composed of blocks of $8 \times 8$ pixels, which can be luminance and/or chrominance blocks $(\mathrm{Cr}+\mathrm{Cb})$. Then the discrete transformed cosine (DTC) is performed on each block to transform them into the frequency domain; the human eye is less sensitive to high frequencies, so the DCT process helps to eliminate or reduce the contribution of these frequency components not detected by the human eye; the low-frequency components detected by the human eye are ordered, quantized, and coded by using a variable-length code (VLC) [21].

MPEG2 also performs temporal processing; it is based on the fact that some areas of a video are repeated several times in different frames during a given period of time. Therefore, MPEG2, instead of coding the same block again and again, saves a reference, past or future, of the block that is repeated and does not send information from blocks that have already been sent; subsequently, this greatly increases compressibility [21]. MPEG2 also performs an algorithm known as motion compensation prediction where the algorithm recognizes block groups based on their luminance components to detect moving objects from frame to frame and, thus, avoids coding the object information in each frame by sending only the spatial movement information, taking as reference a previous or future frame.

To do this, MPEG defines three types of frames: (1) Table I, there are pictures where only spatial and not temporal compression is performed, so they can be completely reconstructed with the information contained without relying on other tables. (2) Table $\mathrm{P}$ contains only the information that changed from Tables I or P immediately preceding; it depends on the information from previously decoded frames to be reconstructed. (3) Table B has tables that depend on Tables I or P, which precede it or even those that are temporarily in the future with respect to it, the prediction based on future frames requires a rearrangement of the frames before transmission which carries some delay [20] [21].

The advantage of, thus, encoding the pictures can be seen in the amount of data required to represent each frame; usually, a $\mathrm{P}$ frame can have 2 to 4 times less information than Table I on which it is based, a B frame can have even less information than a $\mathrm{P}$ frame by a factor of 2 to 5 times [22].

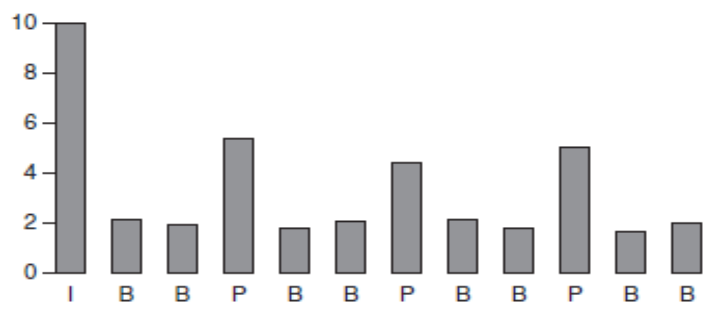

Figure 3. Amount of information concerning the various MPEG tables [22]

A group of pictures (GOP) is a series of pictures that consists of a single I frame and several $\mathrm{P}$ and $\mathrm{B}$ frames; a GOP always starts with an I and ends with the frame previous to frame I from the next GOP Table; all the GOP frames depend directly or indirectly on the initial I-frame [22]. Typically, a GOP has up to $15 \mathrm{P}$ and $\mathrm{B}$ frames, the length is variable and must be chosen carefully because a very short length entails low compression and a very high length raises the complexity and the reproduction of errors.

\subsection{MPEG-4 part 10 - H 264/AVC}

H.264 uses many of the techniques used in MPEG2, but improves them and additionally utilizes numerous amounts of novel compression techniques so compression efficiency with respect to MPEG 2 is notably increased. The most outstanding techniques are described below [20] [23]:

- Multiple frames of reference - frames P and B, which are decoded from other frames are not limited to being referenced only in the preceding frame I or P, but can be referenced from any previously-decoded frame. 


\section{TECCIENCIA}

- Spatial prediction of the macro blocks, based on the adjacent macro blocks, assuming that the difference in luminance among adjacent macro blocks is minimal.

- Variable block size becomes more flexible allowing variable block sizes and with a minimum size of $4 \times 4$ pixels.

- Uses an advanced version of the DCT used in MPEG2.

- More flexible slot size.

- Presents a new ability by partitioning the image into regions called slot groups, where each groove becomes an independently decodable subset.

H.264 is composed of a video coding layer (VCL) and a Network Adaptation Layer (NAL) [24] [25]. The VCL is responsible for implementing the traditional compression techniques at macro block and slot levels. The NAL is considered one of the more novel features of h.264; it is in charge of adapting the bit strings generated by the VCL to various networks and multiplexing environments. NAL achieves this by introducing high-level structures that provide an abstraction at the output of the upper VCL layer. Wiegand et al., [23] explain, in full, the h.264 codec, including all the techniques used and a further explanation of VCL and NAL.

\begin{tabular}{|c|c|c|c|c|c|}
\hline \multirow[b]{2}{*}{ Features } & \multicolumn{5}{|c|}{ Standards (Profiles) } \\
\hline & MPEG-1 & MPEG-2 & $\underset{\mathrm{ASP}}{\text { MPEG-4 }}$ & $\begin{array}{l}\text { MPEG-4 } \\
\text { AVC Main }\end{array}$ & $\begin{array}{c}\text { MPEG-4 } \\
\text { AVC High }\end{array}$ \\
\hline B-Frames & $\checkmark$ & $\checkmark$ & $\checkmark$ & $\checkmark$ & $\checkmark$ \\
\hline Slices Error Resilience & $\checkmark$ & $\checkmark$ & $\checkmark$ & $\checkmark$ & $\checkmark$ \\
\hline Interlace (PAFF/MBAFF] & & $\checkmark$ & $\checkmark$ & $\checkmark$ & $\checkmark$ \\
\hline Entropy Encoding & Huffman & Huffman & Huffman & \begin{tabular}{|c|} 
Exp-Golomb or \\
Adaptive \\
Arithmetic
\end{tabular} & \begin{tabular}{|c|} 
Exp-Golomb ol \\
Adaptive \\
Arithmetic
\end{tabular} \\
\hline Motion Block Size & $16 \times 16$ & $16 \times 16$ & $16 \times 16 / 8 * 8$ & \begin{tabular}{|c|}
$16 \times 16 / 16 \times 8$ \\
$8 \times 8 / 8 \times 4 / 4 \times 4$
\end{tabular} & \begin{tabular}{|c|}
$16 \times 16 / 16 \times 8$ \\
$8 \times 8 / 8 \times 4 / 4 \times 4$
\end{tabular} \\
\hline Intra Prediction & $\begin{array}{l}D C \\
8 \times 8\end{array}$ & $\begin{array}{l}D C \\
8 \times 8\end{array}$ & $\begin{array}{l}\mathrm{AC} \\
8 * 8\end{array}$ & \begin{tabular}{|c|} 
Spatial \\
$16: 16 / 4 \times 4$
\end{tabular} & $\begin{array}{c}\text { Spatial } \\
16 \times 16 / 8 \times 8 \\
\text { or } 4 \times 4\end{array}$ \\
\hline Spatial Transform & $\begin{array}{l}\text { DCT } \\
8 * 8\end{array}$ & $\begin{array}{l}\text { DCT } \\
8 * 8\end{array}$ & $\begin{array}{l}\text { DCT } \\
8 \times 8\end{array}$ & $\begin{array}{l}\text { HCT } \\
4 * 4 \\
\end{array}$ & $\begin{array}{l}\text { HCT } \\
8 \times 884: 4 \\
\end{array}$ \\
\hline Bit Exact Decoding & & & & $\checkmark$ & $\checkmark$ \\
\hline Lossless Mode & & & & & $\checkmark$ \\
\hline Custom Quantizer Matrix & $\checkmark$ & $\checkmark$ & $\checkmark$ & & $\checkmark$ \\
\hline In-loop Deblocking Filter & & & & $\checkmark$ & $\checkmark$ \\
\hline Multiple Reference Frames & & & & $\checkmark$ & $\checkmark$ \\
\hline Weighted Prediction & & & & $\checkmark$ & $\checkmark$ \\
\hline YUV Colour Formats & 4:2:0 & \begin{tabular}{|c|}
$4: 2: 0$ \\
$4: 2: 2,4: 4: 4$
\end{tabular} & 4:2:0 & 4:2:0 & \begin{tabular}{|l|}
$4: 0: 0,4: 2: 0$ \\
$4: 2: 2,4: 4: 4$
\end{tabular} \\
\hline
\end{tabular}

Table 1. Comparison of the major video compression codecs [21]

\section{Transport Protocols in IPV6}

\subsection{MPEG-2 Transport Stream-MPEG2-TS}

When an analog video signal enters an MPEG encoder its output is made up of several independent audio, video, and digital data streams known as "Elementary Streams"; each of these streams is divided into smaller and more manageable packets called "packetized elementary stream" (PES), which may be of fixed or variable length, each PES contains its own header to identify, synchronize and manage each individual stream [21].

Stemming from this PES, MPEG2-TS seeks to create a transport stream that consists of a continuous flow of packets. These packets are formed by breaking PES packets into TS packets of a fixed 188-byte length. Each TS packet consists of 184 data bytes and 4 bytes of header [20]. The main function of MPEG2-TS is to multiplex audio, video, and data and synchronize in the receiver the various streams into one content. Each TS packet contains in its header the PID or packet identifier, which uniquely identifies the Elementary Stream it contains; additionally, each TS packet contains information about the program contained in the form of metadata known as Program-Specific Information (PSI), composed of a series of tables that completely describe the structure of the TS.

\subsection{Real-Time Protocol (RTP) [16]}

The real-time Transport Protocol is a session level protocol defined by the IETF to provide transportation functions over networks that facilitate the delivery of data, like audio and video, in real time, using unicast or multicast techniques [26]. Its most important feature is the ability to carry information regarding the timing of the packets, which allows the receiver to accurately synchronize with the source; RTP also includes a sequence number in its packets, which allows the receiver to estimate how many and which packages have been lost during transportation [21].

The RTP is used to transport up to 7 MPEG2-TS packets in its data field; it uses the UDP transport protocol that provides packet transport not oriented to IP networks connection because UDP is an unreliable protocol; it does not provide recovery of lost packets, but it ensures quick delivery of the data lost during transport. The disadvantage with RTP is that it lacks a mechanism to report the status of the communication, so that it usually relies on the RTCP protocol.

\subsection{Real-Time Transport Control Protocol (RTCP) [16]}

This protocol provides control information regarding the RTP packet stream in a multimedia communication session. With this protocol, the sender and receiver of a session exchange messages 
on the same medium in which the multimedia content is transmitted; in those messages they provide information regarding the status of reception of the contents [27]. Its main function is to provide feedback information regarding the transmission quality of the session from the point of view of the receivers.

\subsection{Real-Time Streaming protocol-RTSP [18]}

It is an application-level protocol that can control the flow of streams of an on-demand video session. It contains functions to start, stop, forward, rewind, or pause playback of multimedia content transmitted by using the RTP protocol, it is important to note that RTSP is not in charge of transporting multimedia but only its control commands [27].

RTSP provides sessions, not connections, so that the transmission does not have to be continuous to maintain a state of connection between the sender and the receiver. RTSP defines a set of messages that allow starting and controlling a session; the most important are described below [27]:

- SETUP: Specifies the transport mechanism used in the session

- DESCRIBE: Obtains the description of a presentation

- PLAY: Used to start or continue the transmission of data to the client. It contains a parameter called scale used to forward or rewind the video stream at different speeds

- RECORD: It is used to start recording the data on the server

- PAUSE: Temporarily pause the content. If applied to the video, the playback is paused; if applied only to the audio it serves to mute the sound keeping the video in playback.

\subsection{Independent Multicast Protocol (IMP)}

It is a family of multicast protocols for IP networks, allowing the configuration and distribution of IP packets on multicast tree structures. IMP itself does not contain any mechanism to discover the topology of the network; instead, it uses the routing information from other traditional protocols like BGP, RIP, OSPF to create its own multicast routing table [28].

IMP - Dense Mode or IMP-DM [29] is a variant of this protocol, it operates on the principle of periodically flooding a data network to build a "tree" of all potential users, and then remove users who do not want to belong to the tree. Every three minutes the flooding process is repeated to discover new users; it is the fastest process for forming a multicast "tree", but at the cost of increasing network congestion [30].

IMP - Sparse Mode and IMP-SM [31], the most widely used protocol for IPTV services; IMP-SM creates a single multicast tree focused on a router known as "Rendezvous Point" or RP, for all multicast users [32]. To create the tree, interested users send messages through a multicast group management protocol like IGMP or MLD.

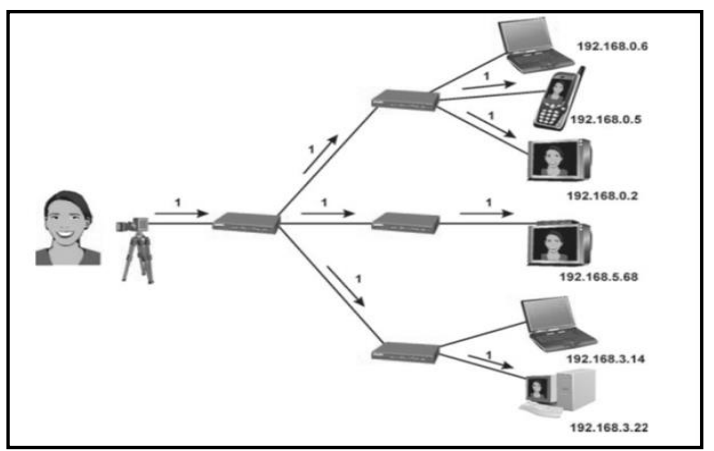

Figure 4. Creating a multicast tree for IPTV traffic [33]

4.6 Multicast Listen Discovery (MLD) [34] [35]

When users wish to join a multicast group to receive information from a specific multicast address, they must use a multicast group management protocol. In IPv4, this protocol is called IGMP and three versions are available; for IPv6, the MLD protocol is used. MLD is used by an IPv6 router to discover the presence of multicast users in links directly connected to it and also to discover what multicast addresses are of interest to those users [36]. This information is transmitted to the multicast routing protocol (IMP-SM) used by the router to ensure that multicast packets are delivered to all links where there are interested users.

MLD uses three different types of ICMPv6 messages, (1) Query messages are used to identify the multicast addresses owned by users in the router's interfaces and can also be used to send to a specific address to learn more about which online users connected to the router interfaces listen to that address, (2) Report messages generally respond to query messages with the multicast IPv6 address you want to hear, (3) Done messages are sent when a user wants to stop listening to a specific multicast address, it sends within this message to the router the multicast address they want to stop hearing [20]. 


\section{TECCIENCIA}

\section{IPTV system based on IMSC}

The IP Multimedia Subsystem (IMS) was introduced by 3GPP [9] as an architectural subsystem dedicated to control and provide multimedia services over packet-based core networks based within third generation mobile networks [37]. Although IMS was created to run within mobile networks, it presented some concepts so novel regarding the way of controlling multimedia services that it was adopted by the NGN architecture, which is based on providing a unique architecture that provides multimedia services to different types of terminals, both fixed and mobile.

Adopting IMS, NGN acquires a control subsystem that allows standardized and controlled communication with various devices using the SIP protocol [38]. Using IMS facilitates convergence of networks, easily enabling communication among different types of networks (MPLS, PSTN, UMTS, WiFi).

IPTV as a standardization service has similarly adopted an architecture based on NGN and IMS, which has been quite successful. In [39] the IPTV platform is defined based on IMS as a service architecture, which allows providing IPTV services controlled and monitored by the IMS subsystem and delivered regardless of the transport network used. The main function of IMS within IPTV is to allow users to discover the entire range of IPTV services offered by the network and perform the authorization and authentication of users on the network.

\subsection{Advantages of using IMS in IPTV [37] [38] [39]}

- Registration and authentication of users in integrated manner in the IMS core network

- Management of user subscription

- Establishment and management of the communication session using SIP

- Control of the QoS

- Personalization of services through a centralized user's database management

- Convergence of fixed and mobile networks

\subsection{Functional Architecture}

Figure 3 shows the functional architecture of the IPTV service based on IMS, as defined by the ETSIVTISPAN. It consists of different functional blocks, each of which complies with specific functions. The service discovery functions (SDF) and service selection functions (SSF) provide the information requested by the user equipment (UE) to select an IPTV service; SDF enables acquiring the information to establish the service connection and SSF offers users personalized information regarding the service [39].

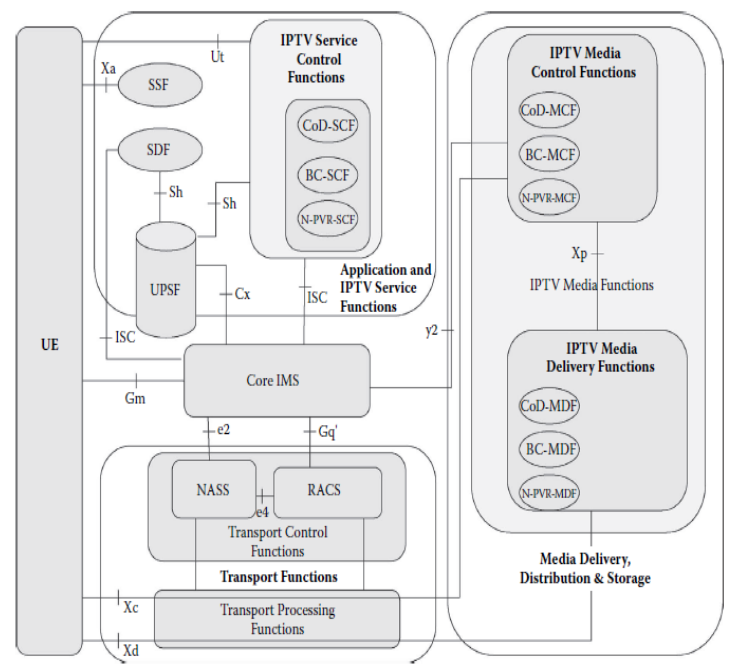

Figure 5. Functional architecture of the IMS-based IPTV system [40]

The service control function (SCF) is responsible for responding to requests for service initiation based on the protocol to start SIP sessions, which are delivered through the IMS Core for any kind of IPTV service requested by the UE [41]. Multimedia control functions (MCF) and multimedia delivery functions (MDF) have a master-slave relationship where $\mathrm{MCF}$ is responsible for controlling the signaling with the SCF through the IMS Core, enabling multimedia connections, while MDF manages the delivery of content to users and reports its status to the MCF [42].

In the transport layer, two subsystems are defined by the NGN architecture: the subsystem of coupling to the mesh network (NASS) responsible for providing IP addresses and other settings to the UE; and the resources control and recognition (RACS) subsystem responsible for booking and allocating resources for multimedia transmission [43].

In the middle between the service and the transport layer is the IMS Core, the center of all the IMS architecture and through which all the signaling and initiation of service messages pass.

\subsection{IMS Core}

The IMS core is used to carry all SIP signaling used in IMS networks to control communication [41] sessions; it is important to clarify that only signaling messages, mainly based on the SIP Protocol transit through the IMS Core, although there are some interfaces that communicate through other protocols 
such as Diameter [44] or XCAP [45]; the traffic flow of multimedia sessions, as is in the case of IPTV channels, is not allowed through the IMS Core.

The IMS Core should not be confused with the transport network core; they are two different elements with different functions. The IMS Core defines a set of session call control functions (CSCF), which are responsible for establishing the multimedia session with (and among) subscribers, and to prepare for the delivery of services requested according to the characteristics of the session requested by users. The IMS Core is composed of four main components described in [14]- [39].

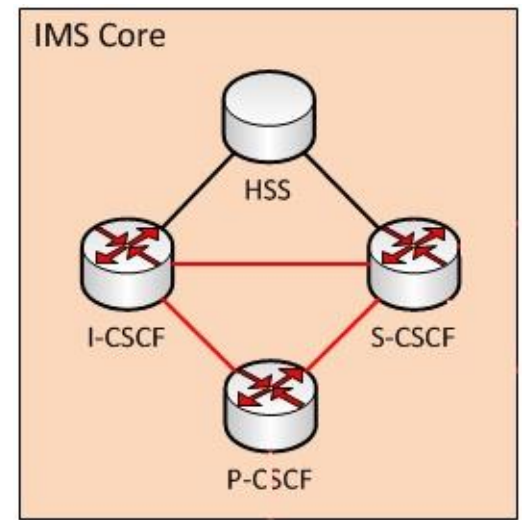

Figure 6. IMS Core and its components [Source: created by authors]

- Proxy-CSCF (P-CSCF): It is the first meeting point for users. Its main function is to ensure the delivery of signaling messages among networks and users; in addition to reserving resources for multimedia delivery

- Serving-CSCF (S-CSCF): it is the main control entity in the IMS system. It processes user registrations and stores their current location; it is responsible for user authentication and session management.

- Interrogating-CSCF (I-CSCF): Checks the user database (HSS) to find the appropriate S-CSCF for each user.

- Home Subscriber Server (HSS): is a central database that contains all the information related to user subscription, stores user profiles and policies that must be implemented by the S-CSCF for each user in personalized manner.

\section{Conclusions and future work}

IPTV has emerged as a new technology to broadcast television content over packet networks based on the IP protocol; due to it, interactive services can be provided to renew the way we will watch and enjoy TV in the future. Studying the architecture, components, and protocols that enable the development of an IPTV infrastructure is important and it is the main intention of this article, as well as studying the most innovative and latest technologies that allow deploying an IPTV service with the easily accessible technology in existence today.

In future work, the aim is to implement a television transmission system prototype operating through IP networks, using IPv6 network addressing as the technology that will dominate networks around the world. In the prototype, the plan is to implement an architecture based on IMS due to the great acceptance that it has had by all major standardization entities and the advantages that implementing such a system entails.

\section{Acknowledgments}

This work was supported and partly carried out at the Center for High Performance Computing (CECAD) at Universidad "Francisco José de Caldas". We thank both COLCIENCIAS and its "Young Researchers" program for supporting the development of this research.

\section{References}

[1] D. Wood, V. Stoss, L. Chan Lizardo, G. Papacostas y M. Stinson, «Virtual Private Networks,» de International Conference on private Switching Systems and networks, 1988.

[2] International Telecommunication Union ITU, «Agentia Nationala Pentru Reglementare,» January 1998. [En línea]. Available: http://www.anrceti.md/files/filefield/Recomandarea\%20 ITU\%20H.323_0.pdf. [Último acceso: 06 April 2015].

[3] Committed to connecting the world, «Telecommunication Sandarization Sector,» ITU, [En línea]. Available: http://www.itu.int/en/ITUT/Pages/default.aspx. [Último acceso: 06 April 2015].

[4] I. K. Park, O. Seung Hun, Y. S. Kwon y H. Young Song , «An implementation of user-participated interactive IPTV service system,» de IEEE International Symposium on cosmenr electronics, Perth, 2008.

[5] K. Kerpez, D. Waring, G. Lapiotis, J. Lyles y R. Vaidyanathan, «IPTV service assurance,» IEEE Communications Magazine, vol. 44, n 9, pp. 166-172, 2006

[6] H. Zeadally, H. Moustafa y F. Siddiqui, «Internet Protocol television (IPTV),» Architecture, trends and challenges, Systems journal, vol. 5, no 5, pp. 518-527, 2011.

[7] G. Myoung, L. Chae, S. Lee, W. Seop Rhee y J. Kyun Choi, «Functional architecture for NGN-based prersonalized IPTV services,» IEEE Transactions on Boradcasting, vol. 55, $\mathrm{n}^{\circ}$ 2, pp. 329-342, 2009.

[8] Eurepean Telecommunications Standards Institute, «Telecommunications and Internet Converged services and protocols for advanced networking,» Sophia Antipolis, France, [En línea]. Available: www.etsi.org/tispan. [Último acceso: 07 April 2015]. 


\section{TECCIENCIA}

[9] 3GPP, «The Mobile Boradband Standard,» 3GPP, [En línea]. Available: www.3gpp.org. [Último acceso: 07 April 2015]

[10] IPTV, «IPTV Forum,» [En línea]. Available: www.iptvforum.com.

[11] ITU-T International Telecommunication Union, «Series Y: Global Information Infrastructure, Internet Protoco aspects and Next-generation Networks. "IPTV Functiona architecture",» september 2008. [En línea]. Available: http://webcache.googleusercontent.com/search?q=cache: B7FkktBInc0J:https://www.itu.int/rec/dologin_pub.asp \%3Flang\%3De\%26id\%3DT-REC-Y.1910-200809I!!PDF-E\%26type\%3Ditems+\&cd=1\&hl=es419\&ct=clnk\&gl=co. [Último acceso: 07 April 2015].

[12] Telecommunication standardization sector of ITU, «Series Y: Global information infrastructure, internet protocol aspects and next- generation networks "Functional requirements and architecture of the NGN release 1" Y. 2012,» International telecommunication Union. ITU-T, 2006.

[13] W. Geng, W. Lenan y W. Deguo, «The technical framework of End-to- END video transmission system for the IPTV,» de 2nd IEEE International Conference on Information Management and Engineering, Beijing, 2010

[14] M. A. Qadeer y A. H. Khan, «Multimedia Distribution over IPTV and its Integration with IMS,» de International conference on data storage and data engineering DSDE, Bangalore, 2010.

[15] E. Rosen, A. Viswanathan y R. Callon, «Multiprotoco Label Switching Architecture,» IETF RFC 3031, 2001.

[16] H. Schulzrinne, S. Casner, R. Frederick y V. Jacobson, «RTP: A Transport Protocol for real-time Aplications,» IETF RFC 3550, 2003.

[17] J. Postel, «User datagram protocol,» RFC 768, 1980.

[18] H. Schulzrinne, A. Rao y R. Lanphier, «Real Time streaming protocol,» IETF RFC 2326, 1998.

[19] MPEG, «The MPEG home page,» [En línea]. Available: http://mpeg.chiariglione.org/.

[20] G. O'Driscoll, Next generation IPTV services and technologies, New York: Wiley, 2008.

[21] J. Francisco, J. Hens y M. Caballero, Triple play: Building the converged Network for IP, VoIp and IPTV, Wiley, 2008.

[22] W. Simpson, Video over Ip. IPTV internet video. Web Tv and streaming, a complete guide to understanding the technology, New York: Focal Press, 2008.

[23] G. J. S. G. B. A. L. T. Wiegand, «Overview of the H.264/AVC Video coding standar,» Circuits and Systems for Video, vol. 13, $\mathrm{n}^{\circ}$ 7, pp. 560-576, 2003

[24] A. Argyriou y V. Madisetti, «Streaming h 264/avc video over the internet,» de Consumer communications and networking conference, Las Vegas, 2004.

[25] S. Wenge, «H.264/VC over IP,» Circuits and Systems for Video, vol. 13, no 7, pp. 645-656, 2003.

[26] g. Hield, Uniderstanding IPTV, New York: Aurebach Publications, 2006

[27] J. Hjelm, Why IPTV: interactivity, technologies, services, New York: Wiley, 2008

[28] M. Taufiqul Islam y A. Hoque, «Study of Reliable Multicast for IPTV Service,» 11 August 2008. [En línea] Available:
http://www.researchgate.net/publication/242230095_Stu dy_of_Reliable_Multicast_for_IPTV_Service.

[29] A. Adams, J. Nicholas y W. Siadak, «Protocol Independent Multicast - Dense Mode (PIM-DM) Protocol Specification,» IETF RFC 3973, 2005.

[30] L. Harte, Introduction to Data Multicasting, IP Multicas Streaming for Audio and Video Media Distribution, Slough: Althos, 2008

[31] B. Fenner, M. Handley, H. Holbrook y I. Kouvelas, «Protocol Independent Multicast - Sparse Mode (PIMSM): Protocol Specification,» IETF RFC 4601, 2006.

[32] A. Quadir, M. Arefin y H. Sandström, « Reliable IPTV Service Delivery Using PIM-SSM Routing,» Journal of Scientific Research, vol. 1, nº 3, 2009.

[33] A. Punchihewa, «Tutorial on IPTV and its latest developments,» de 5th International Conference on Information and Automation for Sustainability (ICIAFs), 2010.

[34] S. Deering, W. Fenner y B. Haberman, «Multicast Listener Discovery for IPv6,» IETF RFC 2710, 1999.

[35] R. Vida y L. Costa, «Multicast Listener Discovery Version 2 (MLDv2) for IPv6,» IETF RFC 3810, 2004

[36] D. Minoli, IP Multicast with Applications to IPTV and Mobile DVB-H, Wiley-IEEE , 2008

[37] E. Mikoczy, D. Sivchenko, B. Xu y J. I. Moreno, «IPTV systems, standards and architectures-part II: IPTV services over IMS: architecture and standardization,» IEEE Communications Magazine, vol. 46, $\mathrm{n}^{\circ} 3$, p. pp $128-135,2008$

[38] J. Rosenberg, H. Schulzrinne, G. Camarillo y A. Johnston, « SIP: Session Initiation Protocol,» IETF RFC 3261, 2002

[39] E. Mikoczy, D. Sivchenko, B. Xu y V. Rakocevic, «IMS based IPTV services: architecture and implementation", de Proceedings of the 3rd international conference on Mobile multimedia communications , 2007.

[40] O. Friedrich, S. Arbanowski, A. Al-Hezmi y R. Seeliger, IMS-Based IPTV IP Multimedia Subsystem Handbook, CRC Press, 2009.

[41] E. Mikoczy, S. Schumann, P. Podhradsky, T. Koski y M. Heinikangas, «Hybrid IPTV Services with IMS Integration of IMS based IPTV with Broadcast and Unicast Mobile TV Services Using DVB-H,» de Fifth International Conference on Next Generation Mobile Appl, Cardiff, 2011.

[42] M. Tsietsi, R. Musvibe, A. Terzol y G. Wells, «Toward IPTV Service Discovery and Selection in an IMS Environment,» de International Congress on Ultra Modern Telecommunications and Control Systems and Workshops (ICUMT), Moscow, 2010.

[43] M. Zahid, M. Qadeer y A. Iqbal, «Deployment of IPTV over IMS architecture,» de 2nd International Conference on Internet Multimedia Services Architecture and Applications, 2008.

[44] P. Calhoun, J. Loughney y E. Guttman, «Diameter Base Protocol,» IETF RFC 3588, 2003.

[45] J. Rosenberg, «The Extensible Markup Language (XML) - Configuration Access Protocol (XCAP),» IETF RFC 4825, 2007 